\title{
Moldagem convencional $x$ Moldagem digital: onde estamos e para onde vamos
}

\section{Conventional impression $x$ Digital impression: where we are and where we are going}

\author{
Moldeo convencional $\mathbf{x}$ Moldeo digital: dónde estamos y hacia dónde vamos
}

\author{
Isabella Cardoso Filgueiras Gomes ${ }^{1 *}$, Carlos Roberto Teixeira Rodrigues ${ }^{2}$, Cezar Romero Furtado \\ Teixeira $^{3}$, Marcelo Vieira Bruno ${ }^{4}$.
}

Como citar esse artigo. Gomes, ICF; Rodrigues, CRT; Teixeira, CRF; Bruno, MV. Moldagem convencional x Moldagem digital: onde estamos e para onde vamos. Revista Pró-UniverSUS. 2021 Jan./Jun.; 12 (1): 54-59.

\begin{abstract}
Resumo
Uma boa moldagem é fundamental para o sucesso do processo reabilitador. O uso da técnica convencional utilizando o silicone de adição ainda se faz presente em muitos consultórios, pois é confiável e precisa. Porém com as inovações e avanços tecnológicos na odontologia, a técnica de impressão digital vem sendo aperfeiçoada há mais de 30 anos. Necessita-se saber se esta técnica atingiu os mesmos níveis de precisões e confiabilidades que a convencional. Este trabalho tem como objetivo, comparar a moldagem convencional com silicone de adição e a moldagem digital, quanto à precisão, adaptação marginal e biossegurança, assim realizou-se uma revisão de literatura utilizando as bases de dados PUBMED, SCIELO E BVS através das palavras-chave: "CAD/CAM", "impressão convencional", "impressão digital", "impressão de arco total", "silicone de adição" e "precisão". O resultado da análise mostrou que, embora os materiais de impressão convencionais possuam ótima precisão, eles possuem algumas desvantagens, como desconforto para o paciente, causam muita sujeira, possuem várias etapas que possibilitam maior risco de causar imperfeições, e exigem uma maior experiência do profissional, enquanto as impressões digitais são mais confortáveis para os pacientes, mais ágeis e mais fáceis de usar. Ambas as técnicas de impressões digitais e convencionais demonstraram ser capazes de produzir resultados clinicamente aceitáveis, então os cirurgiões dentistas deverão basear a sua escolha de acordo com o conceito que melhor se adapta à sua prática clínica atual e o trabalho que deseja realizar. Palavras-chave: Técnica de Moldagem Odontológica; Tecnologia Odontológica; Precisão da Medição Dimensional; Biossegurança; CAD/CAM.
\end{abstract}

\begin{abstract}
A good impression is fundamental for the success of the rehabilitation process. The use of the conventional technique using the addition silicone is still present in many offices, as it is reliable and accurate. However, with innovations and technological advances in dentistry, the digital printing technique has been perfected for more than 30 years. It is necessary to know if this technique has reached the same levels of precision and reliability as the conventional one. This work aims to compare conventional molding with addition silicone and digital molding, in terms of precision, marginal adaptation and biosafety, so a literature review was carried out using the PUBMED, SCIELO AND BVS databases through the keywords- key: "CAD / CAM", "conventional printing", "digital printing", "full arc printing", "addition silicone" and "precision". The result of the analysis showed that, although conventional printing materials have excellent accuracy, they have some disadvantages, such as discomfort for the patient, cause a lot of dirt, have several steps that allow a greater risk of causing imperfections, and require a greater professional experience, while fingerprints are more comfortable for patients, more nimble and easier to use. Both fingerprint and conventional techniques have been shown to be capable of producing clinically acceptable results, so dental surgeons should base their choice on the concept that best suits their current clinical practice and the work they wish to do.

Keywords: Dental Impression Technique; Technology Dental; Dimensional Measurement Accuracy; Biosafety; CAD/ CAM.
\end{abstract}

${ }^{1 *}$ Acadêmica no Curso de Odontologia, Universidade de Vassouras. Vassouras, RJ, Brasil. E-mail: bellafilgueiras28@gmail.com ORCID: https://orcid.org/0000-0002-2753-8295

${ }^{2}$ Doutor. Docente do Curso de Odontologia, Universidade de Vassouras. Vassouras, RJ, Brasil. E-mail: rodriguescrt@gmail.com ORCID: https://orcid.org/0000-0001-6218-1706 ${ }^{3}$ Docente do Curso de Odontologia, Universidade de Vassouras. Vassouras, RJ, Brasil. E-mail: cezar.teixeira@universidadedevassouras.edu.br ORCID: https://orcid.org/0000-00019888-1675

${ }^{4}$ Docente do Curso de Odontologia, Universidade de Vassouras. Vassouras, RJ, Brasil. E-mail: mvieirabruno@gmail.com ORCID: https://orcid.org/0000-0002-9375-0868 


\section{Resumen}

Una buena impresión es fundamental para el éxito del proceso de rehabilitación. El uso de la técnica convencional con silicona de adición todavía está presente en muchas oficinas, ya que es confiable y precisa. Sin embargo, con las innovaciones y avances tecnológicos en odontología, la técnica de impresión digital se ha perfeccionado durante más de 30 años. Es necesario saber si esta técnica ha alcanzado los mismos niveles de precisión y fiabilidad que la convencional. Este trabajo tiene como objetivo comparar el moldeo convencional con silicona de adición y el moldeo digital, en términos de precisión, adaptación marginal y bioseguridad, por lo que se realizó una revisión de la literatura utilizando las bases de datos PUBMED, SCIELO Y BVS a través de las palabras clave- clave: "CAD / CAM", "impresión convencional", "impresión digital", "impresión de arco completo", "silicona de adición” y "precisión”. El resultado del análisis mostró que, si bien los materiales de impresión convencionales tienen una excelente precisión, tienen algunas desventajas, como incomodidad para el paciente, provocan mucha suciedad, tienen varios pasos que permiten un mayor riesgo de causar imperfecciones, y requieren una mayor experiencia profesional. mientras que las huellas dactilares son más cómodas para los pacientes, más ágiles y fáciles de usar. Se ha demostrado que tanto las técnicas de huellas dactilares como las convencionales son capaces de producir resultados clínicamente aceptables, por lo que los cirujanos dentales deben basar su elección en el concepto que mejor se adapte a su práctica clínica actual y al trabajo que desean realizar.

Palabras clave: Técnica de Moldeo Dental; Tecnología dental; Precisión de Medición Dimensional; bioseguridad; CAD/ CAM.

\section{Introdução}

Amoldagem é um procedimento muito importante na área odontológica, pois é através dela que se obtém o molde utilizado no tratamento reabilitador. Ela deve reproduzir, de forma negativa, a anatomia e tamanho real dos dentes, tecidos bucais e estruturas adjacentes. A partir desse molde negativo é possível a obtenção do modelo, forma positiva. ${ }^{1}$

Há pouco tempo existia no mercado odontológico apenas a moldagem convencional, feita com materiais pastosos ou semifluidos que se tornam rígidos ou elásticos após o tempo de presa. ${ }^{1}$

Para que uma boa moldagem seja feita, o material utilizado deve ser atóxico, ter tempo de trabalho satisfatório, hidrofílicos, possuir estabilidade dimensional, bom reprodutor de detalhes e compatível com produtos utilizados para esterilização. ${ }^{2}$

Dentro dos materiais disponíveis para a moldagem convencional, o silicone de adição se destaca por se encaixar nas características necessárias para uma boa reprodução. O polivinilsiloxano tem sido utilizado desde a meados da década de 70 e se mantém até os dias atuais na área odontológica com sua característica principal, a estabilidade dimensional. ${ }^{3}$

Com a constante evolução dos sistemas tecnológicos, novos materiais e tecnologias têm sido criadas e aprimoradas para facilitar e permitir uma prática odontológica com maior qualidade. Uma das inovações que permitem isso é o scanner intraoral com o sistema de impressões digitais CAD/CAM. ${ }^{4}$

A evolução dos scanners intraorais fez com que o fluxo de trabalho se tornasse mais eficiente na área odontológica. A moldagem digital pode ser feita de duas maneiras, direta ou indiretamente. Na forma direta há necessidade do scanner intraoral, onde as informações que são adquiridas possibilitam que o computador gere um modelo e na forma indireta é utilizado o scanner de bancada, que faz a captura através de modelos de gesso ou direto da própria moldagem. ${ }^{5}$

Mesmo com esses avanços e melhorias ainda há dados limitados sobre a precisão das impressões digitais que têm sido produzidas com diversos scanners. O que indica que ainda há necessidade de evidências científicas nesse campo e a dúvida se realmente a substituição da moldagem convencional pela digital é vantajosa. ${ }^{6,7}$

O objetivo deste trabalho é, através de uma revisão de literatura, comparar a moldagem convencional com silicone de adição e a moldagem digital, quanto à precisão, adaptação marginal e biossegurança.

\section{Materiais e Métodos}

Os artigos foram pesquisados nas bases de dados PubMed, Scielo e Bireme com os descritores "CAD/CAM", "impressão convencional", "impressão digital", "impressão de arco total", "silicone de adição" e "precisão" nos idiomas português e inglês, coleta em 2020.

\section{Resultados e Discussão}

A precisão da moldagem é fundamental para que o tratamento odontológico reabilitador seja executado da melhor forma, trazendo os melhores resultados para o paciente. Estudos comparativos e revisões sistemáticas foram feitas entre a precisão da técnica de impressão digital e impressão convencional utilizando como material de moldagem o silicone de adição.

Um estudo in-vitro comparando a precisão das moldagens convencional e digital, realizando a impressão de um modelo de referência de arco completo. As impressões obtidas através de ambas as moldagens foram comparadas com o modelo de referência, através de um scanner de referência altamente preciso. Chegando ao resultado de que a impressão feita pelo método digital revelaram maiores desvios quanto à precisão, já 
o método convencional se mostrou altamente preciso. ${ }^{8}$

Através do estudo onde um molde de arco completo superior com dentes feitos de material cerâmico, foi usado como referência para obter os modelos através da moldagem convencional, utilizando o silicone de adição, e moldagem digital, a fim de analisar a precisão do arco completo e do arco parcial. As impressões foram repetidas 10 vezes para cada grupo. As impressões convencionais foram vazadas com gesso tipo IV e digitalizadas com um scanner de laboratório (inEos X5). Todos os conjuntos de dados foram cortados em áreas de arco completo (dente 17 ao 27), segmento anterior (dente 14 ao 24) ou segmento posterior (dente 13 ao 17) para a respectiva análise. Para verificar a precisão foi utilizado método e sobreposição tridimensional (3D) com um software especial de análise de diferença 3D (GOM Inspect). Foram encontradas diferenças estatisticamente significantes entre os grupos de teste para métodos de impressão de arco completo e parcial in vitro. O método de impressão convencional mostrou significativamente maior exatidão e precisão do que todos os dispositivos digitais testados na impressão do arco completo. Chegando a conclusão de que dentro das limitações deste estudo, as impressões digitais obtidas pelos scanners intraorais são uma alternativa válida às impressões convencionais para segmentos de arco parcial, no entanto, as impressões de arco completo ainda são desafiadoras para esses dispositivos. Mais estudos in vivo são necessários para apoiar esses resultados. ${ }^{9}$

Um estudo in vivo com objetivo de investigar a precisão de métodos convencionais e digitais para impressões de arco completo. Foram feitas impressões de arco completo utilizando materiais convencionais (poliéter, polivinilsiloxano, hidrocolóide irreversível) e scanners digitais (CEREC Bluecam, CER; CEREC Omnicam, OC; Cadent iTero, ITE; Lava COS, LAV; Lava True Definition Scanner, T-Def; 3Shape Trios, TRI; e 3Shape Trios Color, TRC). As impressões foram repetidas três vezes em cinco participantes e então comparadas dentro e entre os grupos de teste. Chegando ao resultado de que as impressões convencionais mostraram a maior precisão em toda a arcada dentária em todos os grupos, exceto no grupo hidrocolóides irreversíveis. Concluindo que os métodos de impressão convencional e digital apresentam significativa diferença na precisão do arco completo. Os sistemas de impressão digital apresentaram maiores desvios locais dentro do arco completo; no entanto, eles alcançam precisão igual e superior a alguns materiais de impressão convencionais. ${ }^{10}$

Comparando a adaptação marginal de coroas de dissilicato de lítio fabricadas a partir da técnica de impressão convencional (silicona de adição) e da técnica de impressão digital (Lava C.O.S.). Utilizado como modelo uma maxila completa feito de zircônia com um preparo no segundo pré-molar direito. As impressões realizadas pela técnica convencional (Aquasil Ultra, Dentsply Caulk) foram vazadas com gesso tipo IV, e então feito um enceramento no dente 15 , após foram confeccionadas 15 coroas de dissilicato de lítio (IPS e.max Press, Ivoclar Vivadent). As impressões digitais foram realizadas pelo sistema Lava C.O.S. e a partir do planejamento virtual (Core3dcentres) foram confeccionadas 15 coroas de dissilicato de lítio (IPS e.max CAD, Ivoclar Vivadent) feitas a partir de uma fresadora (DMG20, Mori Seiki). Foi avaliada a desadaptação marginal entre o preparo e a coroa em 8 pontos diferentes da margem circunferencial através de imagens feitas por um microscópio de alta precisão (Edmund E-Zoom, Edmund Optics Inc). Foi utilizado a análise de variância (método ANOVA) para comparar diferenças estatisticamente significativas entre os grupos. A média da desadaptação marginal da técnica digital foi de $48 \mu \mathrm{m}$ enquanto da técnica convencional foi de $74 \mu \mathrm{m}$. Sendo assim, conclui-se que a técnica de impressão digital alcançou valores aceitáveis de precisão clínica e superou a técnica de impressão convencional com resultados mais precisos. ${ }^{11}$

A realização de um estudo in vitro com o objetivo de comparar a precisão de coroas cerâmicas completas que foram obtidas através do escaneamento intraoral utilizando três sistemas, Lava COS (3M ESPE), CEREC (Sirona) e iTero (Straumann) e utilizando duas técnicas convencionais de impressão (silicone de adição um passo e dois passos). Para o teste foi utilizado um modelo de um primeiro molar superior direito simplificado. Foram obtidas 10 impressões de cada técnica e sistema. Os dados obtidos através das impressões digitais foram enviados para um laboratório protético para a confecção de 10 estruturas de cerâmica de acordo com as especificações do fabricante e as impressões das técnicas convencionais de 1 e 2 passos (Express2 Penta Putty/Light Body Standard, 3M ESPE) foram vazadas com gesso tipo IV e escaneadas com um scanner de laboratório (Lava Scan ST). As medidas de precisão foram obtidas por um sistema de medidas 3D (CNC Rapid, Thome Präzision GmbH,Alemanha). Foi avaliada a desadaptação marginal entre o preparo e a coroa em 4 pontos diferentes da margem circunferencial através de imagens feitas por um microscópio de alta precisão (Leitz M420, Leitz, Alemanha). Foi utilizado o método ANOVA para comparar diferenças estatisticamente significativas entre os grupos. Quanto à adaptação interna, os melhores resultados foram obtidos pelo sistema digital Lava C.O.S. (29 $\mu \mathrm{m}$ de desadaptação), seguido pela técnica de impressão convencional de 2 passos $(35 \mu \mathrm{m})$, pela impressão convencional de 1 passo $(36 \mu \mathrm{m})$, pelo sistema iTero $(50 \mu \mathrm{m})$, enquanto os piores resultados foram obtidos pelo sistema digital CEREC Bluecam ( $88 \mu \mathrm{m})$. Apenas o CEREC Bluecam mostrou diferença estatisticamente significativa para as 
técnicas convencionais. Quanto à adaptação marginal, os melhores resultados foram do CEREC Bluecam (30 $\mu \mathrm{m})$, seguido pela impressão convencional de 1 passo $(33 \mu \mathrm{m})$, pelo sistema iTero $(41 \mu \mathrm{m})$, pelo sistema Lava C.O.S. $(48 \mu \mathrm{m})$, e por último a impressão convencional de 2 passos $(60 \mu \mathrm{m})$. Apenas a técnica convencional de 2 passos teve diferença estatisticamente significativa para as outras técnicas. Concluindo que os dois métodos de impressão (digital e convencional) alcançaram níveis aceitáveis de precisão para a prática clínica. ${ }^{12}$

Um estudo com o objetivo de avaliar os possíveis benefícios e a eficácia das técnicas de impressão digital e convencional. Foram selecionadas dezessete referências do PubMed, nove do Web of Science e uma da central Cochrane de Registros. Com base nos títulos, resumos e texto completo, dezesseis artigos foram selecionados do PubMed, nove do Web of Science e um da central Cochrane. O número total de artigos foi reduzido a dezenove devido alguns estudos estarem repetidos em dois bancos de dados. Nos dados obtidos foi possível chegar aos seguintes resultados: ambas as técnicas de impressão (digital e convencional) possuem o mesmo nível de na fabricação de coroas e pequenas próteses parciais fixas, sendo o silicone, o material utilizado para a moldagem convencional nos artigos consultados. Para a fabricação de coroas suportadas por implantes, a precisão das impressões digitais foi clinicamente aceitável. Nas impressões de arco completo, os métodos deimpressãoconvencional possuemumamelhorprecisão quando comparado ao método digital. Conclui-se que as impressões digitais são uma alternativa clinicamente aceitável como método de impressão para fabricação de coroas e pequenas próteses parciais fixas. Para a fabricação de coroas implanto-suportadas e próteses fixas o método digital também apresentam resultados clinicamente aceitável em relação a adaptação. As impressões digitais são mais rápidas e podem reduzir o tempo de operação total deste processo. Com base nesses estudos, também chegou à conclusão que as impressões convencionais continuam sendo o melhor método para impressões de arco completo. ${ }^{13}$

As siliconas de adição, também conhecidas como polivinilsiloxana, são elastômeros à base de borracha. Começaram a ser produzidas e comercializadas em 1975 e são compostas de uma pasta densa (granulação grossa) e pasta fluida (granulação fina) cuja mistura se dá por adição. ${ }^{3}, 14,15,16$

Nenhum subproduto volátil é liberado durante a reação de polimerização, por esse fato, o silicone de adição é o material de moldagem mais estável e sem a necessidade de ser vazada com gesso imediatamente, possibilitando que o molde seja guardado por uma semana e vazado até três vezes sem alterações. ${ }^{17,18,19}$

O silicone de adição é hidrofílico, portanto, tem capacidade de absorção de líquidos, o que permite boas moldagens em nível gengival e após a polimerização torna-se resistente à sorção de líquidos e são facilmente desinfetadas com líquidos desinfetantes como Glutaraldeído a $2 \%$ ou Hipoclorito de sódio $0,5 \%$ ou $1,0 \% .^{20,21}$

As siliconas de adição são, entre os materiais de moldagem convencional, o mais preciso do mercado, com resistência ao rasgamento, menor alteração dimensional, bom tempo de trabalho e ótima recuperação elástica. Porém possui desvantagens como o alto custo entre os demais, contaminação com sangue e saliva e desconforto do paciente durante a moldagem., 21,22

A sigla $\mathrm{CAD} / \mathrm{CAM}$ do termo inglês compurter aided designer/computer aided manufacturing, em tradução para o português significa desenho auxiliado por computação e manufatura auxiliada por computação. É um sistema que permite de forma mais avançada à confecção de modelos e próteses tridimensionais de última geração. ${ }^{23}$

Este sistema foi desenvolvido pela indústria de aviação e automotiva. Começou a ser incluído na odontologia no final da década de 70 e início de 80, sendo os países pioneiros os Estados Unidos, França e Suíça. O primeiro a ser utilizado e comercializado no meio odontológico foi o Cerec, desenvolvido por Mormann e Brandestini em 1987. Posteriormente novos sistemas foram desenvolvidos como o sistema Procera, Lava, E-Max, Zirkonzahn e All Ceram. ${ }^{24,25,26}$

Os primeiros scanners desenvolvidos empregavam a tecnologia estática de fotografia digital e necessitavam da utilização de dióxido de titânio em pó como contraste. O único objetivo era obter dados para a produção de uma única restauração, sem integração com outros fluxos de trabalho. Os scanners manipulados atualmente, a captura de imagem é feita na velocidade da taxa de vídeo e no geral não necessita do uso de contraste. A aquisição desses dados digitais pode ser utilizada não somente para fabricar restaurações únicas, mas também restaurações de arco completo. ${ }^{27}$

$\mathrm{O}$ uso desse sistema tecnológico simplifica, automatiza e garante altos níveis de qualidade das reabilitações. Ele possui três componentes essenciais: o scanner que faz a leitura e aquisição da imagem, o CAD que é o software de desenho e o CAM que é o sistema de fresagem da estrutura. A obtenção do modelo é através da tecnologia de prototipagem rápida não necessitando do vazamento em gesso. ${ }^{10,23,25,28}$

As impressões digitais têm como vantagens o conforto do paciente, a visualização em tempo real, dados mais fáceis de serem armazenados, digitalização automática de cores, maior eficiência de tempo e a fácil transferência de dados digitais para melhor comunicação entre o profissional e o paciente. Porém este sistema demanda de conhecimento e técnicas avançadas que ainda muitos profissionais não adquiriram, além do alto custo para a aquisição dessa tecnologia. Em pacientes edêntulos totais, onde não há indicação para implantes, $o$ 
uso dos scanners digitais representa um desafio, pois há falta de densidade de dados e incapacidade de capturar uma impressão funcional. ${ }^{27,29,30}$

A obtenção de precisão durante uma impressão é um dos maiores desafios na odontologia, exigindo o domínio do cirurgião dentista sobre a técnica utilizada. A técnica convencional exige mais experiência e é mais difícil de dominar que a técnica de impressão digital. Outro fator considerado, é a intolerância de alguns pacientes quanto aos materiais de impressão convencionais, muitos sentem desconforto quando o material é colocado em boca. ${ }^{31}$

Em estudos realizados, o scanner intraoral apresenta impressão altamente precisa apenas em pequenas áreas, como, somente, um preparo dentário ou um quadrante. Entretanto para impressões de arco completo, os mesmos autores relatam que a moldagem com um material de alta precisão, como o silicone de adição, fornece impressão mais precisas. ${ }^{10,13}$

Quanto a adaptação marginal, a desadaptação marginal da técnica digital com o sistema LAVA C.O.S foi de $48 \mu \mathrm{m}$, enquanto o da técnica convencional foi de $74 \mu \mathrm{m}$, obtendo o resultado de que a técnica de impressão digital alcançou valores aceitáveis e precisos, e, superou a técnica de impressão convencional.11 Já um estudo in vitro que um dos melhores resultados para a adaptação marginal é o da impressão convencional de 1 passo $(33 \mu \mathrm{m})$ seguido do sistema LAVA C.O.S (48 $\mu \mathrm{m}){ }^{12}$

Outro fator importante para se avaliar além da precisão atualmente, é a desinfecção dos moldes. Com um novo $\beta$-coronavírus (2019-nCoV), sendo transmitido diretamente através de espirro, tosse, inalação de gotículas, salivas, vias oral-fetal e por contato com a mucosa oral, nasal e ocular, os cirurgiõesdentistas, auxiliares, protéticos e estudantes dessa área são diariamente expostos ao risco de infecção por esse vírus, devido à grande exposição aos fluidos, sangue e saliva e o manuseio de instrumentais perfurocortantes. O protocolo de biossegurança exerce papel fundamental contra a contaminação cruzada. ${ }^{32}$

Todo o processo de moldagem envolve contato com o paciente e riscos biológicos, principalmente nas moldagens convencionais quando comparadas aos escaneamentos intraorais, por esses fatos a desinfecção dos moldes obtidos devem ser realizadas de acordo com o protocolo de cada material de moldagem. Portanto o escaneamento intraoral pode ser um grande aliado no controle da biossegurança. ${ }^{32}$

\section{Conclusão}

A partir da literatura consultada pode-se concluir que a etapa da moldagemé um dos principais desafios para os cirurgiões-dentistas, que as siliconas polimerizadas por adição são os materiais de moldagem convencionais mais precisos e estáveis e que ambas as técnicas de impressões digitais e convencionais demonstraram ser capazes de produzir resultados clinicamente aceitáveis, possuindo tanto vantagens como desvantagens, então os cirurgiões dentistas deverão basear a sua escolha de acordo com o conceito que melhor se adapta à sua prática clínica atual e o trabalho que deseja realizar.

\section{Referências Bibliográficas}

1. Shilingburg HT, Hobo S, Whitsett LD, Jacobi R, Brackett SE. Fundamentos de prótese fixa. 4.ed. São Paulo: Quintessence; 2007.

2. Pegoraro LF, do Valle AL, dos Reis Pereira de Araujo C, Bonfante G, Conti PCR. Prótese Fixa: bases para o planejamento em reabilitação oral. 2.ed. São Paulo: Artes Médicas; 2013.

3. Brown D. Factors affecting the dimensional stability of elastic impression materials. Journal of Dentistry. 1973 Agosto;1(6): 265-274. DOI https://doi.org/10.1016/0300-5712(73)90103-6.

4. Ueda NC. Sistema CAD/CAM como ferramenta na odontologia: revisão de literatura. (Monografia)- Faculdade de Odontologia, Universidade Estadual de Londrina, Londrina, 2015.

5. Ender A., Mehl A. Accuracy in Dental Medicine, A New Way to Measure Trueness and Precision. J. Vis. Exp. 29 de abril de 2014 (86). doi:10.3791/51374.

6. Chiu A, Chen YW, Hayashi J, Sadr A. Accuracy of CAD/CAM Digital Impressions with Different Intraoral Scanner Parameters. Sensors (Basel). 20 de fevereiro de 2020; 20 (4): 1157. PubMed; PMID: 32093174. 4.

7. Ender A, Zimmermann M, Mehl A. Accuracy of complete- and partialarch impressions of actual intraoral scanning systems in vitro. Int J Comput Dent. 2019[acesso em 16 de março de 2020]; 22(1): 11-19. Disponível em https://ijcd.quintessenz.de/ijcd_2019_01_s0011.pdf3

8. Ender A, Mehl A. In-vitro evaluation of the accuracy of conventional and digital methods of obtaining full-arch dental impressions. Quintessence Int. 2015;46(1):9-17.

9. Ender A, Zimmermann M, Mehl A. Accuracy of complete- and partialarch impressions of actual intraoral scanning systems in vitro. International Journal of Computerized Dentistry. 2019;22(1):11-19.

10. Ender A, Attin T, Mehl A. In vivo precision of conventional and digital methods of obtaining complete-arch dental impressions. J. Prosthet. Dent., St. Louis, v. 115, no. 3, p. 313- 320, Mar. 2016.

11. NG, J.; Ruse, D.; Wyatt, C. A comparison of the marginal fit of crowns fabricated with digital and conventional methods. J. Prosthet. Dent., St. Louis, v. 112, no. 3, p. 555-560, Sept. 2014.

12. Seelbach P, Brueckel, C, Wostmann, B. Accuracy of digital and conventional impression techniques and workflow. Clin. Oral Investig., Berlin, v. 17 , no. 7 , p. 1759-1764, Sept. 2013

13. Ahlholm P, Sipila K, Vallittu P, Jakonen M, Kotiranta U. Digital Versus Conventional Impressions in Fixed Prosthodontics: A Review. J Prosthodont. 2018; 27(1):35-41. Disponível em:https://onlinelibrary.wiley. com/doi/full/10.1111/jopr.12527

14. Valle AL. Moldagem e modelo de trabalho. In: Pegoraro LF, Valle AL, Araujo CRP, Bonfante G, Conti PCR. Prótese Fixa: Bases Para Planejamento Em Reabilitação Oral. 2 ed. São Paulo. Artes Médicas, 2013. 227-267.

15. Acceta DF, Poubel LA. Importância do conhecimento das propriedades de três materiais de moldagem (siliconas e poliéter) - Revisão. Revista Fluminense de Odontologia [Internet]. 2010 [acesso 2020 fevereiro 20];34: 55-59. Disponível em: https://periodicos.uff.br/ijosd/article/ view/30379/17614

16. Chaing BKP. Polymers in the service prosthethic dentistry. J Dent. 1984 setembro; 12(3):203-214. DOI https://doi.org/10.1016/0300- 


\section{2(84)90063-0}

17. Anusavice K.J. Philips: materiais dentários. Rio de Janeiro: Elsevier, $2005.764 \mathrm{p}$

18. SHEN C. Materiais de moldagem. In: ANUSAVICE, K. J. Philips: materiais dentários. Rio de Janeiro: Elsevier, 2005. cap. 9, p. 193-238.

19. Sinhoreti MA. et al. Estudo da precisão dimensional de modelos de gesso confeccionados com diferentes técnicas e materiais de moldagem elastoméricos. RFO UPF, Passo Fundo, v. 15, n. 2, p. 139-144, ago. 2010.

20. Garone Netto N, Burger RC. Inlay e Onlay Metálica e Estética $1^{\mathrm{a}}$ ed ,Quintessence, São Paulo, Cap.5, p. 65-71, 1998.

21. Mezzomo H. Suzuki RM. Reabilitação Oral Contemporânea 1a Ed Santos, Cap. 15, p. 657, 2009.

22. SIM JY, et al. Comparing the accuracy (trueness and precision) of models of fixed dental prostheses fabricated by digital and conventional workflows. J. Prosthodont. Res., Tokyo, v. 62, no. 2, p.1-7, Mar. 2018.

23. Thinschert, J, et al. Status of corrente CAD/CAM technology in dental medicine. Int. J. Comput. Dente, New Malden, v.7, n . 1, p. 25-45, Jan 2004

24. Bottino, MA. Percepção: estética em próteses livres de metal em dentes naturais e implantes. São Paulo: Artes Medicas, 2009. 804 p.

25. Correia,A, et al. CAD/CAM: a informática da prótese fixa. Revista odontológica da UNESP, 2006. p. 183-89.

26. Freitas G. Tecnologia CAD-CAM-CNC a serviço da odontologia, 2008. 29 f. Monografia (Pós -graduação em Engenharia de Materiais) Escola de Engenharia, Universidade Federal do Rio Grane do Sul, Porto Alegre, 2008

27. Clinton D, Stevens DDS. Impression-Making in 2020: How Long Before Analog Methods Are Obsolete? Compedium of Continuing Education in Dentistry [Internet]. 2020 [acesso em 2020 Apr 15];41(3) Disponível em: https://www aegisdentalnetwork.com/cced/2020/03/impression-making-in2020-how-long-before-analog-methods-are-obsolete?token=wLhWYqGawi QJLR2F90QzwixND

28. Wildgoose D, Johnson A, Winstanley RB. Glass/ceramic/refractory techniques, their development and introduction into dentistry: A historical literature review. J. Prosthet. Dent., St. Louis, v. 91, no. 2, p.136-143, Fev. 2004

29. Yuzbasioglu E, Kurt H, Turunc R, Bilir H. Comparison of conventional and digital printing techniques: Evaluation of patients perception, comfort of treatment, efficacy and clinical results. BMC Oral Health.2014, 14(10).

30. Punj A, Bompolaki D, Garaicoa, J. Dental impression materials and techniques. Dente. Clin. N. Am. 2017, 61, 779-796.

31. Lee, S. J.; Macarthur, R. X. TH; Gallucci, G. O. An evaluation of student and clinician perception of digital and conventional implant impressions. J. Prosthet. Dent., St. Louis, v. 110, no. 5, p. 420-423, Nov. 2013.

32. Peng X, Xu X, Li Y, Cheng L, Zhou X, Ren B. Transmission routes of 2019-nCoV and controls in dental practice. Int J Oral Sci. 2020;12(1):9. Published 2020 Mar 3. doi:10.1038/s41368-020-0075-9 